\title{
Reviewing on convexification methods of AC optimal power flow

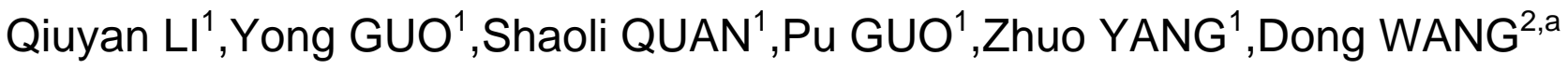 \\ ${ }^{1}$ State Grid Henan Economic Research Institute, Zhengzhou, 450052, China \\ ${ }^{2}$ State Grid Zhumadian Power Supply Company, Zhumadian, 463000, China aemail:1186448369@qq.com
}

Keywords: Optimal Power Flow; Convexification Methods;

\begin{abstract}
To realize the optimal operation of modern power systems, optimal power flow (OPF) serve as the core mathematical model. Due to the nonlinear and nonconvex nature of AC power flow, the solving method of OPF is always attracting ever since its birth. With the development of modern convex optimization developed recently, some novel solving methods have been proposed. To clearly depict the nonconvex characteristics of OPF, two types of AC power flows were reviewed as the theory basis. Then the convexificationmethods, like line programming, quadratic relaxation and so on, were summarized. Finally, the research trend in convexification of AC OPF is given.
\end{abstract}

\section{Introduction}

Power systems are usuallyrequired to operate at least cost or line losses [1].In the viewpoint of mathematics, the operation problem of apower system could be formulated as a here and now nonlinearoptimization problem or called optimal power flow problem(OPF), which determines an equilibrium point correspondingto all operational variables, such as power outputs ofgenerators, shunt capacitor/reactors, voltage values.

An OPF problem optimizes an objective function constrainedby many operational, physical and security constraints, througha set of control variables, as shown in (1).

$$
\begin{array}{r}
\min _{u} f(\mathbf{u}, \mathbf{x}) \\
\text { s.t.g }(\mathbf{u}, \mathbf{x})=0 \\
\mathbf{h}(\mathbf{u}, \mathbf{x}) \leq 0
\end{array}
$$

where $\mathbf{u}$ represents the decision variables, $\mathbf{x}$ stands for the environmental variables, $f(\mathbf{u}, \mathbf{x}), \mathbf{g}(\mathbf{u}, \mathbf{x})$ and $\mathbf{h}(\mathbf{u}, \mathbf{x})$ are the objective function, equality constrains and inequality constrains.

Lotsof researchon OPF has been done since Carpentier's first formulation in 1962 [1-3]. The foundation model in OPF is power flow equations, i.e. the equality contains in (1). These equations use the electrical properties of thetransmission network to relate the real and reactive power injected at each bus to the voltagemagnitude and voltage angle at each bus in a power system. Based on the AC power flows integrated to the OPF models, these models could be classified as bus injection optimal power flows (BIOPF) and branch line optimal power flows (BLOPF).

The classification of control variables, state variables, constrains and objective functions in deterministic OPFs have been well studied in [2]. The main difficulty in solving AC OPFs lies in the non-convexity of power flow equations. The convexity plays an important role in the solving methods robustness, existence of equilibrium in power market and so on.

With modern convex optimization well developed in the past ten years, more and more novel convex relaxation methods for AC OPFs are coming forth. These methods [4-15] could be classified as linearization and quadratic relaxation based on the relaxed model they deployed.

This paper is organized as follows. The bus injection and branch flow models are introduced in Section.2. The convexfication of BIOPF is presented in section 3. Then section 4 reviews the convexfication methods for BFOPF. And the conclusions are given in Section.5. 


\section{AC Power Flow Formulation}

The power flow equations are based on the electrical properties of thetransmission network to relate the real and reactive power injected at each bus. These equations are the integration of the Kirchhofflaws on voltages and currents, the power definition in termsof voltage and current and the power balance at each bus.

Based on the difference of power flow formulation, the most widely deployed models in power flow analysis could be concluded as bus injection flow and branch flow [4]. The above models are both self-contained, which means one could model the power flow by only branch variables or only nodal variables. Then the equivalence between these models is also demonstrated in [4].

\section{Bus injection flow model}

The bus injection model is the standard model for powerflow analysis and optimization, which has been well developed in most commercial software packages. Its most outstanding characteristic is its focus on bus voltage and angle, while indirectly representing the power flow on each line [5].

Within a connected power network graphic $\boldsymbol{G}=(\boldsymbol{N}, \boldsymbol{E})$, where $\boldsymbol{N}:=\{0,1, \ldots, n\}, \boldsymbol{E} \subset \boldsymbol{N} \times \boldsymbol{N}$ and $\mathrm{n}$ is the number of buses, a $(n+1) \times(n+1)$ admittancematrix $\boldsymbol{Y}$ was introduced as follows:

$$
Y_{i j}= \begin{cases}\sum_{k \sim i} y_{i k} & \text { if } i=j \\ -y_{i k} & \text { if } i \neq j \text { jand } i \sim j \\ 0 & \text { otherwise }\end{cases}
$$

where $i \sim j, i \neq j$ jepresents a line in $\boldsymbol{E}$. Further, the graphic $\boldsymbol{G}$ is undirected and $\boldsymbol{Y}$ is symmetric but not necessary Hermitian.

Then the bus injection flow is formulated as:

Kirchhoff law: $\mathbf{I}=\mathbf{Y} \boldsymbol{V}$

Power definition: $S_{i}=V_{i} I_{I}^{*}, i \in N$.

Power balance: $s_{i}=-S_{i}, i \in N$.

where $V_{i}, I_{i}$ and $S_{i}$ are the complex voltage at bus $i$, complex current andcomplex power injections from bus $i$ to the reset of the networks; $s_{i}$ is the complex power absorptionat bus $i$ (load minus generation). Obviously, the power flow on each line is not formulated.

As shown in above model, the state variables are only voltage magnitude and voltage angle at each bus $\{\boldsymbol{V}, \boldsymbol{s}\}$, the line flow are output variables as shown in the following functions[6]:

$$
\begin{aligned}
& p_{i j}=g_{i j} V_{i}^{2}-g_{i j} V_{i} V_{j} \cos \left(\theta_{i}-\theta_{j}\right)-b_{i j} V_{i} V_{j} \sin \left(\theta_{i}-\theta_{j}\right)(6) \\
& q_{i j}=-b_{i j} V_{i}^{2}+b_{i j} V_{i} V_{j} \cos \left(\theta_{i}-\theta_{j}\right)-g_{i j} V_{i} V_{j} \sin \left(\theta_{i}-\theta_{j}\right)(7)
\end{aligned}
$$

where $p_{i j}$ and $q_{i j}$ denote the active and reactive power flow online $i \sim j \in \boldsymbol{E}$, respectively; $\theta_{i}$ is the voltage angle at bus $i ; b_{i j}$ and $g_{i j}$ are the conductance and the susceptance of line $i \sim j \in \boldsymbol{E}$, respectively.

The rectangular form of (6)-(7) could be represented as following:

$$
\begin{aligned}
& p_{i j}=g_{i j}\left(e_{i}^{2}+f_{i}^{2}\right)-g_{i j}\left(e_{i} e_{j}+f_{i} f_{j}\right)-b_{i j}\left(e_{j} f_{i}-e_{i} f_{j}\right) \\
& q_{i j}=-b_{i j}\left(e_{i}^{2}+f_{i}^{2}\right)+b_{i j}\left(e_{i} e_{j}+f_{i} f_{j}\right)-g_{i j}\left(e_{j} f_{i}-e_{i} f_{j}\right)
\end{aligned}
$$

where $e_{i}=V_{i} \cos \left(\theta_{i}\right)$ and $e_{i}=V_{i} \sin \left(\theta_{i}\right)$.

Then the other integrated kind form of Kirchhoff law(3) and power balance (5) could be represented as following:

$$
s_{i}+\sum_{i \sim j}\left(p_{i j}+j q_{i j}\right)=0, i \in N
$$

Then (8)-(10) is the other kind form of bus injection flow.

\section{Branch flow model}

In the branch flow model, a $(n+1) \times$ mincidence matrixCrepresenting the network topology could be defined as following, where $m$ is the number of branches: 


$$
C_{i e}=\left\{\begin{array}{l}
1 \quad \text { if line } e \in \boldsymbol{E} \text { leaves node } i \in N \\
-1 \text { if line } e \in \boldsymbol{E} \text { enters node } i \in N \\
0 \quad \text { otherwise }
\end{array}\right.
$$

where $e$ represents one line belongs to the set of lines $\boldsymbol{E}$.

Contraryto bus injection flow, $G$ in branch flow is a directed graph. Then the Kirchhoff law, power definition, and power balance related to branch flow could be modeled as following [4]:

$$
\begin{aligned}
& \text { Kirchhoff law: } \boldsymbol{I}=\boldsymbol{Z}^{-1} \boldsymbol{C}^{t} \boldsymbol{V} \\
& \text { Power definition: } \\
& \qquad S_{i j}=V_{i} I_{i j}^{*},(i, j) \in \boldsymbol{E} \\
& S_{j}=\sum_{i: i \rightarrow j}\left(S_{i j}-Z_{i j}\left|I_{i j}\right|^{2}\right)-\sum_{k: j \rightarrow k} S_{j k}, j \in \boldsymbol{N}
\end{aligned}
$$

where $z_{i j}$ is the impedance of linei $\sim, Z:=\operatorname{diag}\left(Z_{i j}, i \sim j \in \boldsymbol{E}\right), I_{i j}$ and $S_{i j}$ are the complex current and complex power from busto busj.

Then the power flows in the network $\boldsymbol{G}$ could be represented by (12)-(14) with set of variables $\{\boldsymbol{S}, \boldsymbol{V}, \boldsymbol{I}, \boldsymbol{s}\}$. Compared with the bus injection power flow, the branch flow needs more variables, as complex current and power on each line, to representtheelectrical properties in a connected graphic.

\section{Convexification of bus injection optimal power flows}

As shown in above AC power flow equations, due to the power definition, both bus injection andbus power flow models are both nonlinear and nonconvex, resulting in the nonlinear and nonconvex nature of most AC OPF problems. Then in this part the convexification in BIOPFisreviewed.The most widely deployed methods in BIOPF convexification are linearization [7-15], conic relaxation[16-21], semi-definite relaxation[22-24]and so on.

\section{LinearizationRelaxation}

The basic idea of linearization of a multivariate nonlinear function is based on Taylor's series expansion as following [7-8]:

$$
f(x) \approx f\left(x_{0}\right)+f^{\prime}\left(x_{0}\right)\left(x-x_{0}\right)(15)
$$

Then the nonlinear power flow equations are approximately represented by a fixed point $x_{0}$ and its corresponding first order derivation, and this method is well deployed in linearizedpower flow analysis[2], outer approximation[7]. This method is always named as sensitive analysis.As shown in (15), the approximationprecision of linearized techniques applied in BIOPF depends on the fixed point $x_{0}$.It is not applicable when the decision space of $\{\boldsymbol{V}\}$ in bus injection power flows varies within a large range, and single point linearizationmethod mightobtain infeasible solutions of the original problem [2,6].Traditionally, this method was deployed to solve the security constrained OPFs [2], nowadays it has also been applied to model the boundary load flow, affine load flow and OPFs under interval injection uncertainty [8-10].

Another popular way to linearize the power flow equations in OPFs is linearized decoupledpower flow (including DC power flow), which means the voltage magnitude is fixed and only voltage angle is treated as decision variables[2].As this method could only be applied when line conductances are negligible, phase angles across branches are small enough and voltage magnitudes are close to unity anddo not thus affect real power flows[2], this method could only be applied under limited scenario, i.e., power market[10], transmission networks operation optimization[11].Recently, a linear-programming based model which incorporates reactive power and voltagemagnitudes in a linear power flow approximation was proposed in [12]. However, this method could not be directly integrated into the AC OPFs.

As show in (3) and (12), the power definition resulting bilinear $\left(e_{i} e_{j}, f_{i} f_{j}, e_{i} f_{j}, e_{j} f_{i}\right)$ or multi-linear terms $\left(V_{i} V_{j} \cos \left(\theta_{i j}\right), V_{i} V_{j} \sin \left(\theta_{i j}\right)\right)$ in the line flowequalityconstrains(6-9) and the Kirchhoff law is linear in both models.To linear these nonlinearterms, two novel methods have been proposed recently.The most widely used method to reformulate the multi-linear terms iscalled McCormick inequality, which means the multi-linear term is represented by its convex hull.[6,13]Take the $e_{i} e_{j}$ as an example, the McCormick 
inequality for it could be represented as following:

$$
\begin{aligned}
& w \geq e_{\min , i} e_{j}+e_{\min , j} e_{i}-e_{\min , i} e_{\min , j} \\
& w \geq e_{\max , i} e_{j}+e_{\max , j} e_{i}-e_{\max , i} e_{\max , j} \\
& w \leq e_{\min , i} e_{j}+e_{\max , j} e_{i}-e_{\min , i} e_{\max , j} \\
& w \geq e_{\max , i} e_{j}+e_{\min , j} e_{i}-e_{\max , i} e_{\min , j}
\end{aligned}
$$

where $e_{\min , i}$ and $e_{\max , i}$ are the minimum and maximum boundary of $e_{i}$. Since the McCormick inequality couldonly be applied within a narrow range, it is always deployed to formulate the under estimationproblems under spatial branch and bound (s-BB) or alpha branch and bound ( $\alpha$-BB) algorithms for global non-convex optimizationproblems [14].

With additional binary variables introduced, when the rectangle form bus injection flow model (8)-(10) is adopted, the authors in [15] proposed areformulation method based on SOS-2 relaxation to linearize the bilinear terms, further the load characteristics are also taken into consideration. While when the polar form bus injection flow model (6)-(7) is adopted, the cosine and sine functions should also be approximated, and the approximation method could be found in [6].

\section{Quadratic Relaxation}

Power flow equations are quadratic and hence OPF can be formulated as a quadratically constrainedquadratic program (QCQP) [6]. Unlike the linearization relaxation, the power flow nonlinear nature is preserved by introducing conic [16-21] or semi-definite constrains[22-24].

To ease our expression, the QCQP model of OPFs was shown as following [3]:

$\min _{\boldsymbol{V} \in \mathbf{C}^{n}} \mathbf{V}^{t} \mathbf{C}_{0} \mathbf{V}$

s.t. $\mathbf{V}^{t} \mathbf{C}_{m} \mathbf{V} \leq b_{m}, m=1, \ldots, \mathbf{M}$

If $\mathbf{C}_{m}, m=0,1, \ldots, M \in S^{\mathrm{n}}$ are all positive semidefinite, then (17)is a convex QCQP. With an auxiliaryHamiltonrank-1 matrix $\mathrm{W}:=\mathrm{VV}^{\mathrm{t}}$, (17) could be reformulated as following:

$\min _{V \in \mathrm{C}^{n}} \operatorname{trace}\left(\mathbf{C}_{0} \mathbf{W}\right)$

s.t.

$\operatorname{trace}\left(\mathbf{C}_{m} \mathbf{W}\right) \leq b_{m}, m=1, \ldots, M(18)$

$\mathbf{W} \succ 0$

$\operatorname{rank}(\mathbf{W})=1$

Traditionally, this approach is called lifting.

\section{Conic Relaxation}

To our best knowledge, [16] was the early bird in applying conic relaxation to solve the BIOPF. The conic relaxation was firstly applied to overcome the ill condition in radial networks [16], and was deployed to study the static voltagestability analysis in radial networks for further study [17]. The line flow equations(6)-(7) could be reformulated as following:

$$
\begin{aligned}
& p_{i j}=g_{i j} \frac{v_{i}}{\sqrt{2}}-g_{i j} M_{i j}-b_{i j} N_{i j}(19) \\
& q_{i j}=-b_{i j} \frac{v_{i}}{\sqrt{2}}+b_{i j} M_{i j}-g_{i j} N_{i j}(20) \\
& M_{i j}=V_{i} V_{j} \cos \left(\theta_{i}-\theta_{j}\right)(21) \\
& N_{i j}=V_{i} V_{j} \sin \left(\theta_{i}-\theta_{j}\right)(22) \\
& v_{i}=\sqrt{2} V_{i}^{2}, v_{i} \geq 0(23)
\end{aligned}
$$

With the additional constrains shown in (21)-(23), this reformulation is exact in both radial and mesh networks.

Equality constrains (21)-(23) are nonlinearand the feasible region of (19)-(23) is still non-convex. Then the rotate cone is deployed to relax constrains (21)-(22): 


$$
M_{i j}^{2}+N_{i j}^{2} \leq 2 v_{i} v_{j}
$$

Then the feasible region was expanded from the edge of the cycle to the full cycle.The angle information between the additional variables was omitted. Since the conic relaxation was deployed to represent the static electrical properties of thedistributionnetworks, the conic relaxation (19), (20), (23), (24) could not bedirectly applied to transmission networks OPFs [18]-[19], and the following constrains should be integrated:

$$
\tan ^{-1}\left(\frac{N_{i j}}{M_{i j}}\right)=\theta_{i}-\theta_{j}(25)
$$

As (25) is nonlinear, an iterative procedure based on Taylor's series expansion was proposed to linearize equation (25) [20].What's more, constrain (25) was preserved in the later work [21], and the nonlinear model was solved by interior-point method. Then the feasible region of SOCP-BIM-OPF with respect to bus injection power equationscould be represented as following:

$$
\mathrm{F}_{I}(\mathrm{~V})\left\{\mathrm{V}, \mathrm{M}, \mathrm{N} \mid \begin{array}{l}
s_{i}+\sum_{i \sim j}\left(p_{i j}+j q_{i j}\right)=0, i \in \mathrm{N} \\
p_{i j}=g_{i j} \frac{v_{i}}{\sqrt{2}}-g_{i j} M_{i j}-b_{i j} N_{i j}, i \sim j \in \mathrm{E} \\
q_{i j}=-b_{i j} \frac{v_{i}}{\sqrt{2}}+b_{i j} M_{i j}-g_{i j} N_{i j}, i \sim j \in \mathrm{E} \\
M_{i j}^{2}+N_{i j}^{2} \leq 2 v_{i} v_{j}
\end{array}\right\}
$$

\section{Semi-definite Relaxation}

As shown in (19), it is a natural way to omit the last two nonconvex constrains in QCQP-OPF model, and only a linear model is obtained. However, by relaxation the positive constrain $\mathrm{W} \succ 0$, the relaxationwould be nonsense. And retain the $\mathrm{W} \succ 0$, we could obtain the following SDP-BIM-OPF [19]:

$$
\begin{aligned}
& \min _{V \in \mathbf{C}^{n}} \operatorname{trace}\left(\mathbf{C}_{0} \mathbf{W}\right) \\
& \text { s.t. } \\
& \operatorname{trace}\left(\mathbf{C}_{m} \mathbf{W}\right) \leq b_{m}, m=1, \ldots, M \\
& \mathbf{W} \succ 0
\end{aligned}
$$

As far as we know, the SDP-BIM-OPF was firstly introduced in relax the bun injection power model by [19]. The premises for exactness of SDP-BIM-OPFare first studied in [22] with primal-dual SDP solving methods, and a heuristic recovery method is also proposed for the obtained rank-2 solutionsbased on IEEE-test systems similar to the SDP relaxation applied to the QCQPs [23].

The main difficulty of the exactness of SDP-BIM-OPF is how to hold the rank-1 constrain of W.Further based on Theorem 3 provided in [23] the rank-1 constrain of $\mathrm{W}$ could be represented by the following angle cycle regulation conditions:

$$
\sum_{i \sim j \in C}\left(\angle V_{i}-\angle V_{j}\right)=0 \bmod 2 \pi(28)
$$

Since $\mathbf{W}$ is dense in (27), it would increase the computing cost significantly. Exploiting graph sparsityto simplify the SDP relaxation of OPF is first proposed in [24].

Finally, the relationship between conic relaxation and semi-definite relaxation could be found in [3].

\section{Convexification of branch injection optimal power flows}

Since the branch power flow was usually deployed in distribution networks, there are only limited two kinds of methods to reformulate the BFOPF: linearizationmethod and conic relaxation method. As the linearization method is much alike with the linearization techniques deployed in the 
BFOPFs, we would not give the summary here and only the conic relaxation techniques are depicted.

As shown in (12)-(14), the feasible set of BFOPF is complex set. With the following angle relaxation technique, we could transform the complex set to real set [3].

$$
\begin{aligned}
& P_{i}=P_{i j}-r_{i j} l_{i j}-\sum_{k:(j, k) \in E} P_{j k}, \forall j \in N \\
& Q_{i}=Q_{i j}-x_{i j} l_{i j}-\sum_{k:(j, k) \in E} Q_{j k}, \forall j \in N \\
& v_{j}=v_{i}-2\left(r_{i j} P_{i j}+x_{i j} Q_{i j}\right)+\left(r_{i j}^{2}+x_{i j}^{2}\right) l_{i j}, \forall(i, j) \in E(31) \\
& l_{i j}=\frac{P_{i j}^{2}+Q_{i j}^{2}}{v_{i}}, \forall(i, j) \in E(32)
\end{aligned}
$$

As shown in (29)-(32), the only nonlinear and non-convex equal constrains are (32) for each line. If (32) is discarded, a linearized BFOPF could obtained. But the obtained solution would be nonsense, as it could not meet the physical laws in power networks. Then the following rotated conic constrain is introduced as following [25]:

$$
l_{i j} \geq \frac{P_{i j}^{2}+Q_{i j}^{2}}{v_{i}}, \forall(i, j) \in E(33)
$$

And this method was firstly proposed for radial networks, and the exactness premise for the relaxation was also proposed [25]. However, this relaxation could not be hold when applied to mesh grids. So a further angle recovery strategy was proposed with virtual phasorshifter at each line was proposed in [26]. However, this strategy is much more empirical, and should be further studied.

\section{Conclusion}

This paper presents relevant research work applyingconvexificationtechniques for solving the ACOPF problem. It clearly depicts the difficultiesof solving ACOPFs by depicting the widely deployed bus injection flow and branch flow. Then the convexification techniques for both BIOPFs and BFOPF were surveyed and discussed. As much more concerns are paid to distribution networks with increasing penetration of distributed energy resources, the conic relaxation technique for BFOPFscould be more attractive.

\section{Reference}

[1]. Carpentier, J. "Contribution to the economic dispatch problem." Bulletin de la Societe Francoise des Electriciens 3.8 (1962): 431-447.

[2]. Frank, Stephen, Ingrida Steponavice, and Steffen Rebennack. "Optimal power flow: a bibliographic survey I." Energy Systems 3.3 (2012): 259-289.

[3]. Low, Steven H. "Convex relaxation of optimal power flow, Part I: Formulations and equivalence." arXiv preprint arXiv:1405.0766 (2014).

[4]. Subhonmesh, Bose, Steven H. Low, and K. Mani Chandy. "Equivalence of branch flow and bus injection models." (2012): 1893-1899.

[5]. Zhou, Tianshou, et al. "On the optimal solutions for power flow equations." International journal of electrical power \& energy systems 25.7 (2003): 533-541.

[6]. Coffrin, Hassan L. Hijazi Carleton, and Pascal Van Hentenryck. "Convex Quadratic Relaxations for Mixed-Integer Nonlinear Programs in Power Systems."(In press)

[7]. Antonio, J. C., M. Roberto, and C. Enriqne. "Decomposition techniques in mathematical programming." (2006).

[8]. Dimitrovski, Aleksandar, and Kevin Tomsovic. "Boundary load flow solutions." Power Systems, IEEE Transactions on 19.1 (2004): 348-355.

[9]. Mohapatra, A., P. R. Bijwe, and B. K. Panigrahi. "Optimal power flow with multiple data uncertainties." Electric Power Systems Research 95 (2013): 160-167. 
[10]. Haghighat, Hossein, Hossein Seifi, and Ashkan R. Kian. "Gaming analysis in joint energy and spinning reserve markets." Power Systems, IEEE Transactions on 22.4 (2007): 2074-2085.

[11]. González, Pablo, et al. "Joint energy and reserve markets: Current implementations and modeling trends." Electric Power Systems Research 109 (2014): 101-111.

[12]. Coffrin, Carleton, and Pascal Van Hentenryck. "A linear-programming approximation of AC power flows." INFORMS Journal on Computing 26.4 (2014): 718-734.

[13]. McCormick, Garth P. "Computability of global solutions to factorable nonconvex programs: Part I-Convex underestimating problems." Mathematical programming 10.1 (1976): 147-175.

[14]. Gupte, Akshay, et al. "Solving mixed integer bilinear problems using MILP formulations." SIAM Journal on Optimization 23.2 (2013): 721-744.

[15]. Ferreira, Rafael S., Carmen LT Borges, and Mario VF Pereira. "A Flexible Mixed-Integer Linear Programming Approach to the AC Optimal Power Flow in Distribution Systems." (2014): 1-1.

[16]. Jabr, Rabih A. "Radial distribution load flow using conic programming." Power Systems, IEEE Transactions on 21.3 (2006): 1458-1459.

[17]. Jabr, R. A., and B. C. Pal. "Conic programming approach for static voltage stability analysis in radial networks." Generation, Transmission \& Distribution, IET 1.2 (2007): 203-208.

[18]. Taylor, Joshua Adam. Conic optimization of electric power systems. Diss. Massachusetts Institute of Technology, 2011.

[19]. X. Bai, H. Wei, K. Fujisawa, and Y. Wang. Semidefinite programming for optimal power flow problems. Int'l J. of Electrical Power \&Energy Systems, 30(6-7):383-392, 2008.

[20]. Jabr, Rabih A. "A conic quadratic format for the load flow equations of meshed networks." Power Systems, IEEE Transactions on 22.4 (2007): 2285-2286.

[21]. Jabr, Rabih A. "Optimal power flow using an extended conic quadratic formulation." Power Systems, IEEE Transactions on 23.3 (2008): 1000-1008.

[22]. J. Lavaei and S. H. Low. Zero duality gap in optimal power flow problem. IEEE Trans. on Power Systems, 27(1):92-107, February 2012.

[23]. Subhonmesh Bose, Steven H. Low, Thanchanok Teeraratkul, and Babak Hassibi. Equivalent relaxations of optimal power flow. IEEETrans. Automatic Control, 2014.

[24]. X. Bai and H. Wei. A semidefinite programming method with graph partitioning technique for optimal power flow problems. Int'l J. ofElectrical Power \& Energy Systems, 33(7):1309-1314, 2011.

[25]. Li, Na, Lijun Chen, and Steven H. Low. "Exact convex relaxation of OPF for radial networks using branch flow model." SmartGridComm. 2012.

[26]. Farivar, Masoud, and Steven H. Low. "Branch flow model: Relaxations and convexification-Part I." Power Systems, IEEE Transactions on 28.3 (2013): 2554-2564.

[27]. Grossmann, Ignacio E. "Review of nonlinear mixed-integer and disjunctive programming techniques." Optimization and Engineering 3.3 (2002): 227-252. 